\title{
FREDHOLM-VOLTERRA INTEGRAL EQUATION WITH POTENTIAL KERNEL
}

\author{
M. A. ABDOU and A. A. EL-BARY
}

(Received 9 October 2000)

\begin{abstract}
A method is used to solve the Fredholm-Volterra integral equation of the first kind in the space $L_{2}(\Omega) \times C(0, T), \Omega=\left\{(x, y): \sqrt{x^{2}+y^{2}} \leq a\right\}, z=0$, and $T<\infty$. The kernel of the Fredholm integral term considered in the generalized potential form belongs to the class $C([\Omega] \times[\Omega])$, while the kernel of Volterra integral term is a positive and continuous function that belongs to the class $C[0, T]$. Also in this work the solution of Fredholm integral equation of the second and first kind with a potential kernel is discussed. Many interesting cases are derived and established in the paper.
\end{abstract}

2000 Mathematics Subject Classification. 45B05, 45D05, 45E10.

1. Introduction. Many problems of mathematical physics, theory of elasticity, and mixed problems of mechanics of continuous media reduce to an integral equation with a kernel that has one of the following forms:

$$
\begin{aligned}
& K_{n, m}^{\alpha, y}(x, y)=\frac{x^{\alpha}}{y^{\epsilon+\gamma-1}} W_{n, m}^{\alpha}(x, y), \\
& W_{n, m}^{\alpha}(x, y)=\int_{0}^{\infty} \lambda^{\alpha} J_{n}(x \lambda) J_{m}(y \lambda) d \lambda,
\end{aligned}
$$

where $J_{n}(x)$ is a Bessel function of the first kind of order $n$. Arutyunyan [5] has shown that the plane contact problem of the nonlinear theory of plasticity, in its first approximation, can be reduced to Fredholm integral equation of the first kind with Carleman kernel

$$
\begin{aligned}
K_{ \pm 1 / 2, \pm 1 / 2}^{\alpha, 1 / 2}(x, y) & =|x-y|^{-\alpha} \\
& =\sqrt{x y} \int_{0}^{\infty} \lambda^{\alpha} J_{ \pm 1 / 2}(x \lambda) J_{ \pm 1 / 2}(y \lambda) d \lambda, \quad(\varepsilon=0,0 \leq \alpha<1)
\end{aligned}
$$

(for the symmetric and skew symmetric cases, respectively).

In $[14,15]$ Mkhitaryan and Abdou obtained the general formulas, even and odd, of the potential analytic function, using Krein's method [13], for the Fredholm integral equation of the first kind with Carleman kernel [15] and logarithmic kernel [14]

$$
K_{ \pm 1 / 2, \pm 1 / 2}^{0,1 / 2}(x, y)=-\ln |x-y|=\sqrt{x y} \int_{0}^{\infty} J_{ \pm 1 / 2}(x \lambda) J_{ \pm 1 / 2}(y \lambda) d \lambda, \quad(\varepsilon=0)
$$

(for symmetric and skew symmetric, respectively). 
Kovalenko [12] developed the Fredholm integral equation of the first kind for the mechanics mixed problems of continuous media and obtained an approximate solution for the Fredholm integral equation of the first kind with an elliptic kernel

$$
K_{0,0}^{0,1}(x, y)=\frac{2 \sqrt{x y}}{\pi(x+y)} K\left(\frac{\sqrt{2 x y}}{x+y}\right)=\int_{0}^{\infty} J_{0}(x \lambda) J_{0}(y \lambda) d \lambda, \quad(\varepsilon=0) .
$$

Abdou in [1] obtained the solution of Fredholm integral equation of the second kind with potential function kernel, $\left(K(x-\xi, y-\eta)=1 / \sqrt{(x-\xi)^{2}+(y-\eta)^{2}}\right)$,

$$
K_{m, m}^{0,1 / 2}(x, y)=\sqrt{x y} \int_{0}^{\infty} J_{m}(\lambda x) J_{m}(\lambda y) d \lambda, \quad(\varepsilon=0)
$$

Also, in [3], the structure resolvent for the Fredholm integral equation of the second kind with potential function kernel is obtained by Abdou. The potential theory method is used in [4, 2] to obtain the eigenvalues and eigenfunctions for a system of Fredholm integral equations of the first kind with Carleman kernel in [2] and logarithmic kernel in [4]. Abel's theorem is used in [8] to obtain the general solution of the Fredholm integral equation of the first kind with a kernel in the form of the Gauss hypergeometric function

$$
K(x, y)=\frac{1}{\left(x^{2}+y^{2}\right)^{2 n}} F\left(n, n+\frac{1}{2}, m\left(\frac{2 x y}{x^{2}+y^{2}}\right)^{2}\right) .
$$

The solution in Mathieu function form is obtained in [4], where the potential theory method is used for contact problems of mechanics of continuous media between a finite system of stamps with varying width and an elastic half-space in a threedimensional formulation, for which the domain of integration $\Omega$ is represented as $\Omega:(x, y, z) \in \Omega:-\infty<x, y<\infty, z>0$.

In this paper, the solution of Fredholm-Volterra integral equation of the first kind is obtained in $L_{2}(\Omega) \times C(0, T)$, where $\Omega=\left\{(x, y): \sqrt{x^{2}+y^{2}}=r \leq a\right\}, z=0$, and the time $t \in[0, T], T<\infty$. The problem is investigated from the three-dimensional semisymmetric contact problem in the theory of elasticity of frictionless impression of a rigid surface $(G, v)$ having an elastic material occupying the domain $\Omega$, where the external forces are neglected. Assume a function $f(x, y) \in L_{2}(\Omega)$ which describes the surface of stamp, such that, it is impressed into the elastic layer surface (plane) by a variable force $M(t)$, whose rigid displacement $\delta(t) \in C(0, T)$. The integral equation, in this case, becomes (see [1])

$$
\begin{aligned}
& \iint_{\Omega} \frac{P(\xi, \eta, t) d \xi d \eta}{\left[(x-\xi)^{2}+(y-\eta)^{2}\right]^{1 / 2}}+\int_{0}^{t} F(\tau) P(x, y, \tau) d \tau \\
& =\pi \theta[\delta(t)-f(x, y)]=f(x, y, t), \quad\left(\theta=G(1-v)^{-1}\right)
\end{aligned}
$$

under the condition

$$
\iint_{\Omega} P(x, y, t) d x d y=M(t), \quad 0 \leq t \leq T \leq \infty .
$$

Here $F(t)$ is a positive continuous function that belongs to the class $C(0, T)$ and 
represents the characterized resistance of the elastic layer, $P(x, y, t)$ is the unknown potential normal stress function between the surface of stamp and the elastic layer, $G$ is the displacement magnitude and $v$ is Poisson's coefficient.

In this work, the Fredholm integral equations of the first and second kind with a generalized potential kernel are established and their solutions are discussed, the kernel is represented in the Weber-Sonin integral formula. Many interesting spectral relationships are derived from the problem. Finally, a numerical example is considered for the solution of Fredholm integral equation of the second kind.

2. Basic equations. In this section, a method is used to obtain a finite system of integral equations in three dimensions, then, by using the method of separation of variables, we represent the integral equation to a system of Fredholm integral equation of the second kind in one dimension. Also the kernel of Fredholm integral equation is represented in the Weber-Sonin integral formula.

So, we divide the interval $[0, T], 0 \leq t \leq T<\infty$ as $0=t_{0}<t_{1}<t_{2} \cdots<t_{N}=T$, where $t=t_{k} \in[0, T], k=0,1, \ldots, N$; then by using the quadratic formula [6], $u_{j}, j=0,1, \ldots, k$, in the Volterra integral term of (1.2), we have

$$
\int_{0}^{t_{k}} F(\tau) P(x, y, \tau) d \tau=\sum_{j=0}^{k} u_{j} F_{j} P_{j}(x, y)+0\left(\bar{h}^{p+1}\right), \quad\left(h_{k} \longrightarrow 0, p>0\right),
$$

where $\bar{h}=\max _{0 \leq k \leq N} h_{K}, h_{j}=t_{j+1}-t_{j}, P\left(x, y, t_{k}\right)=P_{k}(x, y), F\left(t_{j}\right)=F_{j}$,

$$
u_{j}= \begin{cases}\frac{h}{2}, & j=0, k \\ h, & j \neq 0, k\end{cases}
$$

The values of $u_{j}$ and $p, p \simeq k$, depend on the number of derivatives of $F(t)$ (see [6]). Using (2.1) in (1.2), we have

$$
\begin{aligned}
u_{K} F_{K} P_{K}(x, y) & +\iint_{\Omega} \frac{P_{K}(\xi, \eta) d \xi d \eta}{\left[(x-\xi)^{2}+(y-\eta)^{2}\right]^{1 / 2}}+\sum_{j=0}^{K-1} u_{j} F_{j} P_{j}(x, y) \\
& =\pi \theta\left[\delta_{K}-f(x, y)\right]=f_{K}(x, y), \quad\left(\delta_{K}=\delta\left(t_{K}\right), k=0,1, \ldots, N\right) .
\end{aligned}
$$

Also condition (1.3) becomes

$$
\iint_{\Omega} P(x, y) d x d y=M_{k}, \quad\left(M\left(t_{k}\right)=M_{k}\right) .
$$

The solution of the integral equation (2.3) depends on the kernel and the values of $F_{k}$ at the two points $t_{0}$ and $t_{N}$, for example, if $F\left(t_{0}\right)=F_{0}=0$, the first equation of the linear integral system of (2.3) represents an integral equation of the first kind, then for all values of $k>1$ we have a linear system of integral equations of the second kind, while for $t_{N}=0$, the formula (2.3), for $0 \leq k \leq N-1$, represents a linear system of integral equations of the second kind, and the formula (2.4) at $k=N$, represents an integral equation of the first kind. 
To separate the variables, we assume

$$
\begin{aligned}
& P_{k}(x, y)=P_{k m}(r)\left\{\begin{array}{l}
\cos m \theta, \\
\sin m \theta,
\end{array}\right. \\
& f(x, y)=f_{k m}(r)\left\{\begin{array}{l}
\cos m \theta, \\
\sin m \theta .
\end{array}\right.
\end{aligned}
$$

Using (2.5) in (2.3) and (2.4), we have

$$
\begin{gathered}
u_{k} F_{k} P_{k m}(r)+\int_{0}^{a} \rho W_{m}^{1 / 2}(r, \rho) P_{k m}(\rho) \rho d \rho+\sum_{j=0}^{k-1} u_{j} F_{j} P_{j m}(r)=f_{k m}(r), \\
\int_{0}^{a} \rho P_{k m}(\rho) d \rho= \begin{cases}\frac{M_{k}}{2 \pi}, & m=0, \\
0, & m \geq 1,\end{cases}
\end{gathered}
$$

where

$$
W_{m}^{\alpha}(r, \rho)=\int_{-\pi}^{\pi} \frac{\cos m \phi d \phi}{\left[r^{2}+\rho^{2}-2 r \rho \cos \phi\right]^{\alpha}}, \quad\left(\alpha=\frac{1}{2}+\ell, \ell<\frac{1}{2}\right) .
$$

To write the integral equation (2.8) in the Bessel function form, first we use the following relations [7, page 81$]$ :

$$
\begin{aligned}
& \int_{0}^{2 \pi} \frac{\cos m \phi d \phi}{\left[1-2 z \cos \phi+z^{2}\right]^{\alpha}}=\frac{2 \pi(\alpha)_{m} z^{m}}{m !} F\left(\alpha, m+\alpha, m+1, z^{2}\right), \\
& F\left(\gamma, \gamma+\frac{1}{2}-\beta, \beta+\frac{1}{2}, z^{2}\right) \\
& \quad=(1+z)^{-2 \gamma} F\left(\gamma, \beta ; 2 \beta ; \frac{4 z}{1+z^{2}}\right), \quad\left(|z|<1, \operatorname{Re} \gamma>0,(\gamma)_{m}=\frac{\Gamma(m+\gamma)}{\Gamma(\gamma)}\right) .
\end{aligned}
$$

Hence, (2.8) takes the form

$$
W_{m}^{\alpha}(r, \rho)=\frac{2 \pi \Gamma(m+\alpha)}{m \Gamma(\alpha)} \frac{(r \rho)^{m}}{(r+\rho)^{2 m+1}} F\left(m+\alpha, m+\frac{1}{2}, 2 m+1, \frac{4 r \rho}{(r+\rho)^{2}}\right),
$$

where $F(a, b, c ; z)$ is the Gauss hypergeometric function, and $\Gamma(x)$ is the Gamma function. Formula (2.10) is symmetric and does not depend on the relation between $\rho$ and $r$.

Second, using the relation (see [9])

$$
\begin{aligned}
& \int_{0}^{\infty} J_{\alpha}(a x) J_{\alpha}(b x) x^{-\beta} d x \\
& \quad=\frac{2^{-\beta} a^{\alpha} b^{\alpha} \Gamma(\alpha+(1-\beta / 2))}{(a+b)^{2 \alpha-\beta+1} \Gamma(1+\alpha) \Gamma((1+\beta) / 2)} F\left(\alpha+\frac{1-\beta}{2}, \alpha+\frac{1}{2}, 2 \alpha+1, \frac{4 a b}{(a+b)^{2}}\right),
\end{aligned}
$$

where $J(x)$ is the Bessel function, equation (2.10) takes the form

$$
W_{m}^{1 / 2}(r, \rho)=2 \pi \int_{0}^{\infty} \lambda_{1}^{2 \ell} J_{m}\left(\lambda_{1} \rho\right) J_{m}\left(\lambda_{1} r\right) d \lambda_{1}
$$


Using (2.12) and the following notations:

$$
\begin{gathered}
u=\frac{r}{a}, \quad v=\frac{\rho}{a}, \quad \Phi_{k m}(u)=\frac{P_{k m}(a u)}{\sqrt{a u}}, \quad \lambda=a \lambda_{1}, \quad c^{*}=a^{1+2 \ell} 2 \pi, \\
g_{k m}(u)=\frac{f_{k m}(a u)}{\sqrt{a u}}=\frac{2 \pi \theta}{\sqrt{a u}}\left[\delta_{k}-f_{m}(a u)\right], \quad Q_{k}=\frac{M_{k}}{2 \pi a}, \quad(k=0,1, \ldots, N ; m \geq 0),
\end{gathered}
$$

the integral equation (2.6) and condition (2.7) become

$$
\begin{gathered}
\mu_{k} \Phi_{k m}(u)+\int_{0}^{1} K_{m}^{1 / 2}(u, v) \Phi_{k m}(v) d v+\sum_{j=0}^{k-1} \mu_{j} \Phi_{j m}(u)=g_{k m}(u), \\
\int_{0}^{1} \sqrt{v} \Phi_{k m}(v) d v= \begin{cases}Q_{k}, & m=0, \\
0, & m \geq 1,\end{cases}
\end{gathered}
$$

where

$$
K_{m}^{\alpha}(u, v)=2 \pi a \sqrt{u v} \int_{0}^{\infty} \lambda^{2 \ell} J_{m}(\lambda u) J_{m}(\lambda v) d \lambda
$$

which represents a Weber-Sonin integral formula.

It is easy to prove the following relation:

$$
\left(\frac{\partial^{2}}{\partial u^{2}}-\frac{\partial^{2}}{\partial v^{2}}\right) K_{m}^{1 / 2}(u, v)=(h(u)-h(v)) K_{m}^{1 / 2}(u, v),
$$

where

$$
h(x)=\left(m^{2}-\frac{1}{4}\right) x^{-2}, \quad\left(m \neq \pm \frac{1}{2}\right) .
$$

The integral equation (2.14) represents a linear system of Fredholm integral equation of the first or second kind depending on the values of $\mu_{k}, k \in[0, N]$. The general solution of (2.14) can be obtained using the recurrence relations for values of $k$ and the mathematical induction. For this aim, let $k=0$ in (2.14) and (2.15), we obtain

$$
\mu_{0} \Phi_{0 m}(u)+\int_{0}^{1} K_{m}^{1 / 2}(u, v) \Phi_{0 m}(v) d v=g_{0 m}(u)
$$

under the condition

$$
\int_{0}^{1} \sqrt{v} \Phi_{0 m}(v) d v= \begin{cases}Q_{0}, & m=0 \\ 0, & \text { otherwise. }\end{cases}
$$

The kind and solution of (2.19) depend on the values of $\mu_{0}$, for this, we are going to obtain the solution of (2.19), first when $\mu_{0} \rightarrow 0$ and second when $\mu_{0}$ satisfies the relation

$$
\mu_{0}>\int_{0}^{1} \int_{0}^{1} K_{m}^{1 / 2}(u, v) d u d v, \quad m=0, \pm \frac{1}{2}, \pm 1, \ldots
$$

3. Fredholm integral equation of the first kind. In this section, we obtain the general solution of Fredholm integral equation of the first kind when the kernel takes a Weber-Sonin integral formula and for continuous values of $g_{0 m}(u)$. Also many spectral relationships are established here.

When $F_{0}=0$, we have $\mu_{0}=0$ and (2.19) becomes

$$
\int_{0}^{1} K_{m}^{1 / 2}(u, v) \Phi_{0 m}(v) d v=g_{0 m}(u) .
$$

Abdou in [2] used the potential theory method (see [10]) to solve a linear system of 
Fredholm integral equation in the form of (3.1) under condition (2.20) where the given function is represented in the Jacobi polynomials form. Here, we obtain the solution of (3.1) under condition (2.20) for a given continuous function $g_{0 m}(u)$. For this, rewrite (3.1) and (2.20) as an integral equation of the Wiener-Hopp type [11, 16]. For setting $u=e^{-\xi}, v=e^{-\eta}, e^{-\xi} \Phi_{0 m}\left(e^{-\xi}\right)=\Psi_{m}(\xi)$, and $g_{0 m}\left(e^{-\xi}\right) e^{-\gamma \xi}=h_{m}(\xi)$ in (3.1) and (2.20), we have

$$
\begin{gathered}
\int_{0}^{\infty} M(\xi-\eta) \Psi_{m}(\eta) d \eta=h_{m}(\xi), \quad 0 \leq \xi<\infty, \\
\int_{0}^{\infty} e^{-n / 2} \Psi_{m}(\eta) d \eta= \begin{cases}Q_{0}, & m=0, \\
0, & \text { otherwise }\end{cases}
\end{gathered}
$$

where

$$
M(\xi-\eta)=e^{-\gamma(\xi-\eta)} K_{m}^{\gamma}\left(e^{-\xi}, e^{-\eta}\right) .
$$

Popov [16] stated that, in order to obtain the solution of (3.2) under condition (3.3), it suffices to obtain the most simple equation

$$
\int_{0}^{\infty} M(\xi-\eta) \psi_{z m}(\eta) d \eta=e^{i z \xi}, \quad \xi, \operatorname{Im} z \geq 0 .
$$

Now, making use of the formulae

$$
\Psi_{m}(\xi)=\frac{1}{2 \pi} \int_{-\infty}^{\infty} G(-z) \psi_{z m}(\xi) d z, \quad G(z)=\int_{0}^{\infty} h_{m}(\xi) e^{i \xi z} d \xi .
$$

The solution of $(3.5)$ (see $[5,16])$ is given by

$$
\psi_{z m}(u)=\frac{1}{u} \psi_{z m}\left(\ln \frac{1}{u}\right)=\frac{\psi_{m}^{-}(-z)}{\Gamma(3 / 4)}\left\{\left(1-u^{2}\right)^{-1 / 4}+(m+1+i z) \int_{u}^{1} \frac{t^{-m-2-i z}}{\left(t^{2}-u^{2}\right)^{1 / 4}} d t\right\},
$$

where

$$
\psi_{m}^{-}(-z)=\sqrt{2} \Gamma\left(\frac{1}{2}\left(m+\frac{5}{4}-i z\right)\right)\left(\Gamma\left(m+\frac{1}{2}-i z\right)\right)^{-1} .
$$

After obtaining the solution of (3.7), we can derive the general solution of (3.2). It is easy to see that the function $\sqrt{u} \psi_{z m}(u)$ is a solution of (3.1) when $g_{0 m}(u)=u^{-1-i z}$. Therefore, the general solution of the integral equation

$$
\int_{0}^{1} K_{m}^{1 / 2}(u, v) q_{m}^{1 / 2}(v, 1) d v=1, \quad 0 \leq u<1,
$$

is given by

$$
q_{m}^{1 / 2}(u, 1)=\sqrt{u}\left[\psi_{z m}(u)\right]_{z=i} .
$$

By using the principle of Krein [13], with the aid of (3.10), the general solution of (3.1) takes the form

$$
\begin{aligned}
\Psi_{0 m(u)} & =\frac{\sqrt{2} u^{m+1 / 2}}{\Gamma\left(w^{3 / 4}\right) \Gamma\left(w^{1 / 4}\right)}\left\{\frac{x(1)}{\left(1-u^{2}\right)^{1 / 4}} \int_{u}^{1} \frac{X^{1}(v) d v}{\left(v^{2}-u^{2}\right)^{1 / 4}}\right\}, \\
X(u) & =\frac{u^{-2 m-1 / 2}}{c^{*}} \frac{d}{d u} \int_{0}^{u} \frac{s^{m+1 / 2} g_{0 m}(s) d s}{\left(u^{2}-s^{2}\right)^{1 / 4}}, \quad\left(c^{*}=2 \pi a\right) .
\end{aligned}
$$


Now, we can obtain many interesting cases, for example, replacing $g_{0 m}(u)$ in (3.12) by a Jacobi polynomial, that is, let $g_{0 m}(u)=P_{m}^{(m, 1 / 4)}\left(1-2 u^{2}\right)$, then (3.1) is transformed to

$$
\begin{gathered}
\int_{0}^{1} \frac{u^{1+m} K_{m}^{1 / 2}(u, v) P_{m}^{(m, 1 / 4)}\left(1-2 u^{2}\right) d u}{\left(1-u^{2}\right)^{1 / 4}}=\lambda_{m} v^{m} P_{m}^{(m, 1 / 4)}\left(1-2 v^{2}\right), \\
\lambda_{m}=2^{3 / 2} \Gamma\left(m+\frac{3}{4}\right) \Gamma\left(2 m+\frac{3}{4}\right)[m ! \Gamma(1+2 m)]^{-1}
\end{gathered}
$$

In terms of Gauss hypergeometric function of formula (8) of [14, page 715], we obtain the following important property:

$$
K_{m}^{\alpha}\left(u^{-1}, v^{-1}\right)=(u v)^{\alpha} K_{m}^{\alpha}(u, v) .
$$

Substituting $u=x^{-1}, v=y^{-1}$ in (3.13), and making use of property (3.14), we obtain spectral relations of the semi-infinite interval

$$
\begin{aligned}
& \int_{1}^{\infty} \frac{K_{m}^{1 / 2}(x, y) P_{m}^{(m, 1 / 4)}\left(1-2 y^{-2}\right) d y}{y^{z}\left(y^{2}-1\right)^{1 / 4}} \\
&=\frac{\lambda_{m} P_{m}^{(m, 1 / 4)}\left(1-2 x^{-2}\right)}{x^{3 / 2+m}}, \quad\left(z=\frac{5}{4}+m, 1 \leq x<\infty\right) .
\end{aligned}
$$

4. Fredholm integral equation of the second kind. In this section, the general solution of Fredholm integral equation of the second kind is obtained. Also the mathematical induction is used to obtain the general solution of (2.14) under condition (2.15).

Now, we need to obtain the solution of Fredholm integral equation of the second kind (2.19) under condition (2.20), where its solution depends on the kernel (2.16) and the surface $f_{m}(r)$. When the initial and the tangent points of the surface are in contact with the origin 0 , we can expand $f_{m(u)}$ in Macklorian expansion near $u=0$

$$
f_{m}(u) \cong \frac{f_{m}^{\prime \prime}(0)}{2 !} u^{2}+\frac{f_{m}^{\prime \prime \prime}(0)^{3}}{3 !} u^{3}+\cdots+\frac{f_{m}^{(n)}(0)}{n !} u^{n}+\cdots
$$

Equation (4.1) gives the degree of displacement of the surface for any degree. For example, if the displacement is very small and $f_{m}^{\prime \prime}(0) / 2 !=A_{2} \neq 0$, we obtain $f_{m}(u)=$ $A_{2} u^{2}$.

In general,

$$
f_{m}(u)=A_{2 m} u^{2 m}, \quad A_{2 m}=\frac{f_{(0)}^{(2 m)}}{(2 m) !}, \quad(m \geq 0),
$$

where $m$ is the order harmonic of the contact problem.

Hence, the function $g_{0 m}(u)$ takes the form

$$
g_{0 m}(u)=\left(\Delta_{0}-\beta A_{2 m} u^{2 m}\right) \sqrt{u}, \quad\left(\Delta_{0}=\beta \delta_{0}, \beta=\pi \theta\right) .
$$

Equation (4.3) represents a polynomial of degree $2 m+1 / 2$, and the solution of (2.19) under condition (2.20) depends on the kernel (2.16) and the function (4.3). So, rewrite 
(2.19) and (2.20) to take the following form:

$$
\begin{gathered}
\mu_{0} Z_{m}(u)+\int_{0}^{1} K(u, v) Z_{m}(v) d v=u^{2 m+1 / 2}, \\
\Delta_{0} \int_{0}^{1} \sqrt{u} Z_{0}(u) d u-A_{2 m} \int_{0}^{1} \sqrt{u} Z_{m}(u) d u=Q_{0},
\end{gathered}
$$

where

$$
\Phi_{0 m}(u)=\Delta_{0} Z_{0}(u)-A_{2 m} Z_{m}(u), \quad(m \geq 1) .
$$

To solve (4.4), we use the formula (7.3911) of [9] and with the aid of [2, 8], we can write the kernel (2.16) in the form

$$
K_{m}^{1 / 2}(u, v)=c^{*} \sqrt{2}(u v)^{m+1 / 2} \sum_{j=0}^{\infty} \frac{\Gamma^{2}(j+m+3 / 4) P_{j}^{m}(u) P_{j}^{m}(v)}{\Gamma^{2}(j+1+m)(2 j+m+3 / 4)^{-1}},
$$

where

$$
P_{j}^{m}(u)=P_{j}^{(m, 1 / 4)}\left(1-2 u^{2}\right)
$$

Here $P_{j}^{(m, 1 / 4)}(x)$ is the Jacobi polynomial.

Hence, the solution of (4.4) with the kernel of (4.7) is equivalent to the solution of the linear system

$$
\mu_{0} X_{i}+c^{*} \sum_{j=0}^{\infty} A_{j} B_{i j} X_{j}=f_{i}
$$

where

$$
\begin{aligned}
f_{j} & =\left(2 j+m+\frac{3}{4}\right)^{1 / 4} \int_{0}^{1} f_{m}(u) u^{m+1} P_{j}^{m}(u) d u, \\
A_{j} & =\frac{1}{\sqrt{2}} \frac{\Gamma^{2}(j+m+3 / 4)(2 j+m+3 / 4)^{1 / 4}}{\Gamma^{2}(j+m+1)}, \\
B_{i j} & =\left(2 j+m+\frac{3}{4}\right)\left(2 i+m+\frac{3}{4}\right) \int_{0}^{1} u^{2 m+1} P_{i}^{m}(u) P_{j}^{m}(u) d u .
\end{aligned}
$$

The infinite linear system of (4.9) is solvable under the condition

$$
\sum_{j=0}^{\infty}\left|c^{*} A_{j} B_{i j}\right|<\mu_{0}, \quad\left(c^{*}=2 \pi a\right) .
$$

Using the orthogonality of the Jacobi polynomial, the general solution of (4.4) takes the form

$$
\mu_{0} Z_{m}(u)=u^{2 m+1 / 2}-c^{*} \sum_{j=0}^{\infty} \frac{\sqrt{2} \Gamma^{2}(j+m+3 / 4) u^{m} P_{j}^{m}(u) X_{j}^{m}}{(j+m+1)(2 j+m+3 / 4)^{-3 / 4}} .
$$

Hence by the mathematical induction, the solution of (2.14) can be obtained.

5. Numerical computations. For $j=2, m=3, \mu_{0}=c^{*}=1$, we have the results shown in Table 5.1 
TABLE 5.1

\begin{tabular}{clc}
\hline$u$ & $u^{2 m+1}$ & $Z(u)$ \\
\hline 0.1 & 0.0000 & 0.0000 \\
0.2 & 0.0007 & 0.0009 \\
0.3 & 0.0044 & 0.0045 \\
0.4 & 0.0162 & 0.0156 \\
0.5 & 0.01442 & 0.0415 \\
0.6 & 0.1004 & 0.0937 \\
0.7 & 0.2001 & 0.1883 \\
0.8 & 0.3270 & 0.3096 \\
0.9 & 0.6224 & 0.6070 \\
1.0 & 1.0 & 1.03 \\
\hline
\end{tabular}

6. Conclusions. From the above results and discussions, the following may be concluded.

(1) The three-dimensional semi-symmetric contact problem for a stamp impressed into a layer surface, which was made of material according to the power law $\sigma_{j}=K_{0} \epsilon_{j}$, $j=1,2,3$, by a variable force $N(t)$ represents a Fredholm-Volterra integral equation of the first kind.

(2) The generalized potential kernel represents a Weber-Sonin integral formula

$$
K(u, v)=\sqrt{u v} \int_{0}^{\infty} J_{m}(t u) J_{m}(t v) d t,
$$

which represents a nonhomogeneous wave equation and the kernel can be written in the Legender polynomial form as follows:

$$
K_{m}^{1 / 2}(u, v)=\frac{1}{\sqrt{2}}(u v)^{m+1 / 2} \sum_{n=0}^{\infty} \frac{\Gamma^{2}(n+m+3 / 4) P_{n}^{m}(u) P_{n}^{m}(v)}{\Gamma^{2}(n+m+1)(2 n+m+3 / 4)^{-1}},
$$

where, $P_{n}^{m}(u)$ is a Legendre polynomial.

(3) The Fredholm-Volterra integral equation of the first kind can be reduced to a finite linear system of Fredholm integral equation of the second kind.

(4) This paper is a generalization of the works of the contact problems in continuous media for the Fredholm integral equation of the first and second kind when the kernel takes the following forms: logarithmic kernel, Carleman kernel, elliptic integral kernel, and potential kernel. Moreover, the contact problems that lead to the integrodifferential equation with Cauchy kernel are a special case of (2.19). Also in this work the contact problems of higher-order $(m \geq 1)$ harmonic are included as special cases.

\section{REFERENCES}

[1] M. A. Abdou, Fredholm integral equation of the second kind with potential kernel, J. Comput. Appl. Math. 72 (1996), no. 1, 161-167. MR 97c:45001. Zbl 857.65143.

[2] _ Integral equation and contact problem for a system of impressing stamps, Appl. Math. Comput. 106 (1999), no. 2-3, 141-148. MR 2000g:74059. Zbl 991.37420.

[3] _ Fredholm integral equation with potential kernel and its structure resolvent, Appl. Math. Comput. 107 (2000), no. 2-3, 169-180. MR 2000h:45002. Zbl 991.37400. 
[4] _ Spectral relationships for integral operators in contact problem of impressing stamps, Appl. Math. Comput. 118 (2001), no. 1, 95-111. CMP 1805163.

[5] N. K. Arutyunyan, The plane contact problem of the theory of creep, J. Appl. Math. Mech. 23 (1960), 1283-1313, translated from Prikl. Mat. Mekh. 23(1959), 901-924 (Russian). Zbl 113.39801.

[6] K. E. Atkinson, A Survey of Numerical Methods for the Solution of Fredholm Integral Equations of the Second Kind, SIAM, Pennsylvania, 1976. MR 58\#3577. Zbl 353.65069.

[7] G. Bateman and A. Ergeyli, Higher Transendental Functions, vol. II, McGraw-Hill, New York, 1985, reprint.

[8] M. H. Fahmy, M. A. Abdou, and M. A. Darwish, Integral equations and potential-theoretic type integrals of orthogonal polynomials, J. Comput. Appl. Math. 106 (1999), no. 2, 245-254. MR 2000f:74057. Zbl 929.45004.

[9] I. S. Gradshteyn and I. M. Ryzhik, Table of Integrals, Series, and Products, 5th ed., Academic Press, Massachusetts, 1994, translated from the fourth Russian edition. MR 94g:00008. Zbl 918.65002.

[10] C. D. Green, Integral Equation Methods, Thomas Nelson and Sons, London, 1969. MR 40\#658. Zbl 179.44301.

[11] H. Hochstadt, Integral Equations, Pure and Applied Mathematics, John Wiley and Sons, New York, 1973. MR 52\#11503. Zbl 259.45001.

[12] E. V. Kovalenko, Some approximate methods of solving integral equations of mixed problems, Appl. Math. Mech. 53 (1989), 85-92.

[13] M. Kreĭn, On a method of effective solution of an inverse boundary problem, Doklady Akad. Nauk SSSR (N.S.) 94 (1954), 987-990 (Russian). MR 16,38h.

[14] S. M. Mkhitaryan and M. A. Abdou, On different methods of solution of the integral equation for the planar contact problem of elasticity, Dokl., Akad. Nauk Arm. SSR 89 (1990), no. 2, 69-74 (Russian). Zbl 707.73065.

[15] _ On various methods for solution of the Carleman integral equation, Dokl., Akad. Nauk Arm. SSR 89 (1990), no. 3, 125-129 (Russian). Zbl 709.45006.

[16] G. Y. Popov, Kontaktnye zadachi dlya lineino-deformiruemogo osnovaniya [Contact Problems for a Linearly Deformed Base], Vishcha Shkola, Kiev-Odessa, 1982 (Russian). MR 84d:73004. Zbl 563.73098.

M. A. Abdou: Department of Mathematics, Faculty of Education, Alexandria UniVERSITY, EGYPT

A. A. El-BAry: Department of Basic And Applied Sciences, P.O. BoX 1029 Alexandria, ARAB ACADEMY FOR SCIENCE AND TECHNOLOGY AND MARITIME TRANSPORT, EgyPT

E-mail address: a1aaci ty@hotmai 1.com 


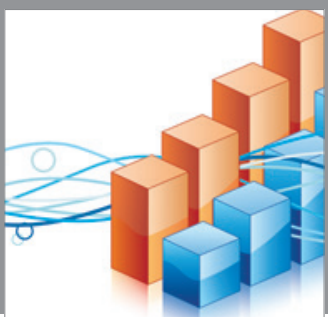

Advances in

Operations Research

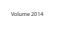

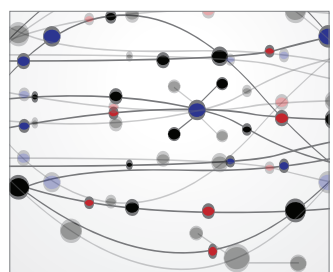

\section{The Scientific} World Journal
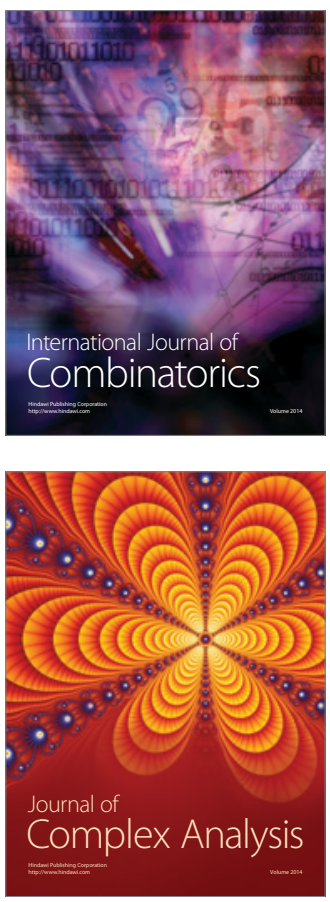

International Journal of

Mathematics and

Mathematical

Sciences
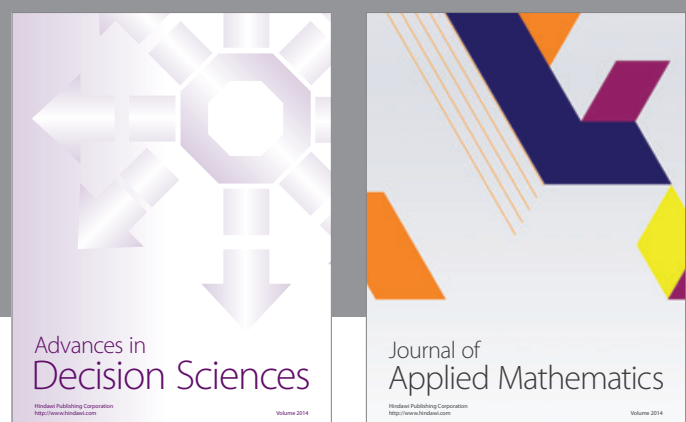

Journal of

Applied Mathematics
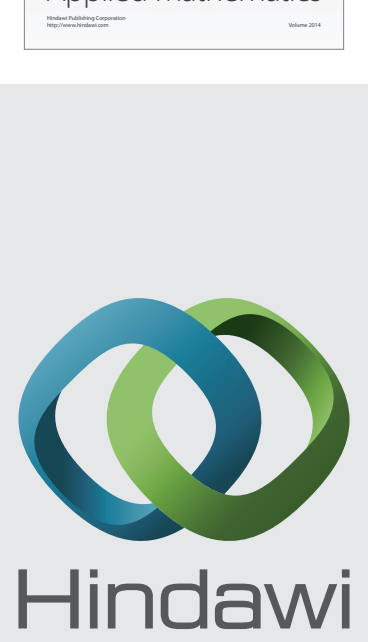

Submit your manuscripts at http://www.hindawi.com
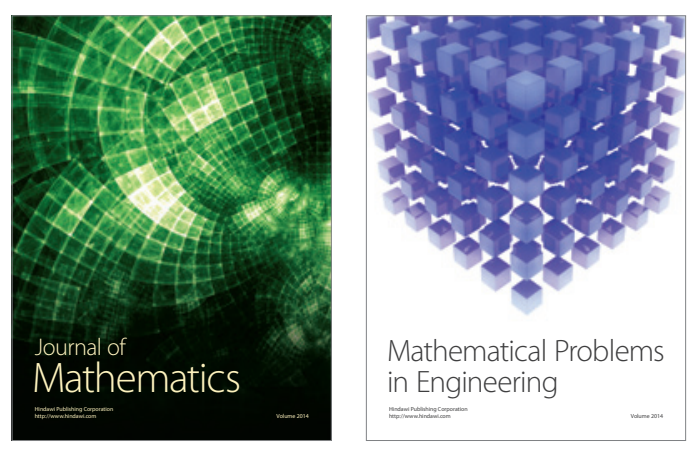

Mathematical Problems in Engineering
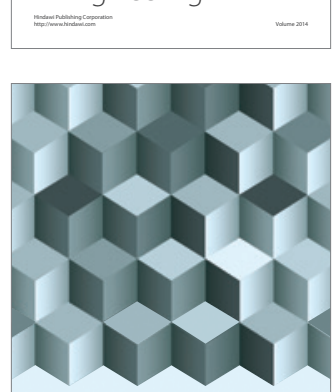

Journal of

Function Spaces
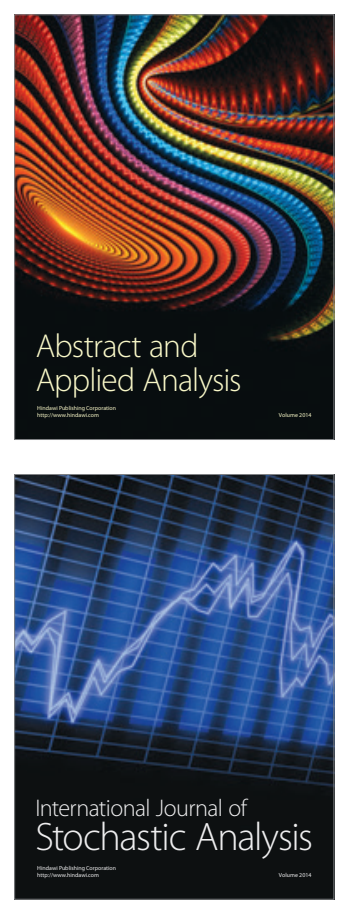

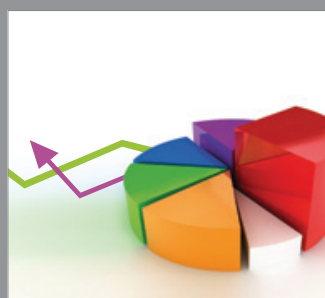

ournal of

Probability and Statistics

Promensencen
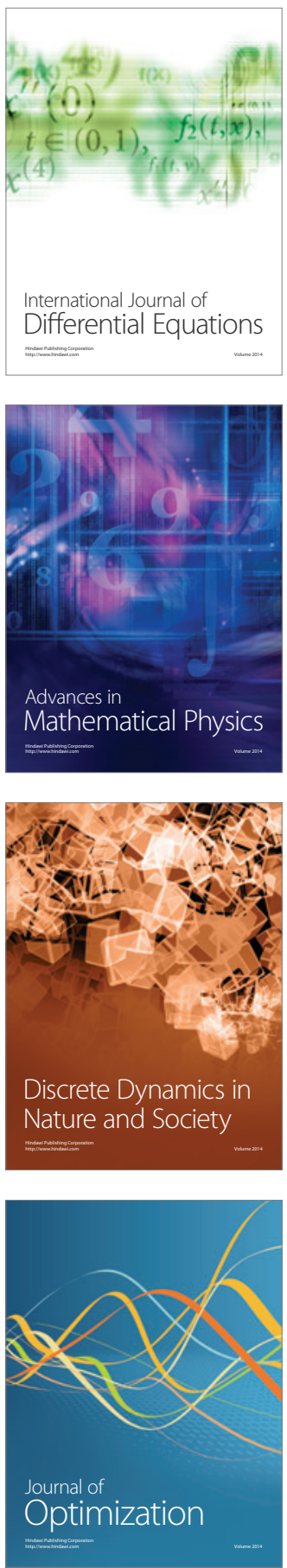